Article

\title{
The Role of Parent-Child Relationships and Filial Expectations in Loneliness Among Older Turkish Migrants
}

\author{
Rowan L. F. ten Kate ${ }^{1, *}$, Başak Bilecen ${ }^{1,2}$ and Nardi Steverink ${ }^{1,3}$ \\ ${ }^{1}$ Department of Sociology, University of Groningen, The Netherlands; E-Mails: r.l.f.ten.kate@rug.nl (R.L.F.T.K), \\ b.bilecen@rug.nl (B.B.), b.j.m.steverink@rug.nl (N.S.) \\ 2 Centre on Migration, Citizenship and Development, Bielefeld University, Germany \\ ${ }^{3}$ Department of Health Psychology, University Medical Centre Groningen, University of Groningen, The Netherlands \\ * Corresponding author
}

Submitted: 12 May 2021 | Accepted: 16 July 2021 | Published: 15 December 2021

\begin{abstract}
Older first-generation migrants living in Europe, particularly Turkish migrants, feel relatively lonely, which indicates social exclusion. Social embeddedness within the family, particularly parent-child relationships, can alleviate loneliness for older migrants, but such relationships can also be ambivalent, which may not prevent loneliness altogether. Earlier research indicates that Turkish migrants in Germany report high quality relationships with their children and high levels of social support exchanges within the family; however, some still report disappointing aspects of the relationship with their children, such as feeling disrespected. To better understand these contradictory findings, this article focuses on various aspects of parent-child relationships that may explain loneliness among older Turkish migrants in Germany. Moreover, the article considers whether filial expectations can be potential sources of intergenerational conflict that may explain higher levels of loneliness among older Turkish migrants. Using the Generations and Gender Survey with 606 first-generation Turkish respondents aged 50 and above, findings show that having low satisfying relationships with children and not having adult co-residing children is associated with more loneliness. Turkish migrants with higher filial expectations feel lonelier when they have good perceived health, and less lonely when they have bad perceived health. These findings indicate that especially healthy older Turkish migrants may have unfulfilled expectations regarding parent-child relationships, which adds to their loneliness, while parents with bad health experience solidarity, which lowers their loneliness. This shows that both intergenerational solidarity and conflict influence loneliness among older Turkish migrants.
\end{abstract}

\section{Keywords}

intergenerational conflict; intergenerational solidarity; intergenerational support; international migration; loneliness; older adults; parent-child relationships

\section{Issue}

This article is part of the issue "In Good Company? Personal Relationships, Network Embeddedness, and Social Inclusion" edited by Miranda J. Lubbers (Autonomous University of Barcelona, Spain).

(C) 2021 by the authors; licensee Cogitatio (Lisbon, Portugal). This article is licensed under a Creative Commons Attribution 4.0 International License (CC BY).

\section{Introduction}

Older first-generation migrants living in Europe, particularly Turkish migrants, feel relatively lonely (van Tilburg \& Fokkema, 2020), which is worrisome given the detrimental impact of loneliness on both mental and physical health (Holt-Lunstad et al., 2015). In some European countries such as Germany, the ageing migrant population from Turkey represents a large migrant group (Steinbach, 2013; van Tilburg \& Fokkema, 2020). Given the increasing numbers of older Turkish migrants who feel relatively lonely, recent research has tried to explain their higher levels of loneliness via factors such as socioeconomic status (SES), health, and feelings of belonging 
(Fokkema \& Naderi, 2013; Klok et al., 2017; van Tilburg \& Fokkema, 2020; Visser \& El Fakiri, 2016). Loneliness is defined as the unpleasant experience when one's social relationships are not as desired in terms of quantity or quality (de Jong Gierveld \& van Tilburg, 2006). Thus, loneliness can be conceptualised as the perception of social inclusion and as the opposite experience of social embeddedness (de Jong Gierveld et al., 2018). As earlier research showed ample evidence of high levels of loneliness among Turkish migrants, this might indicate that they do not feel as socially included as they would like to be. However, the higher feelings of loneliness seem at odds with the previous findings of "strong family cohesion" among Turkish migrants in Europe (BaykaraKrumme \& Fokkema, 2019). This raises the question of whether this family cohesion alleviates overall feelings of social exclusion.

With regards to social relationships, parent-child relationships are particularly central for older adults concerning social support exchanges (Bordone \& de Valk, 2016; Offer \& Fischer, 2018a). In later life, parents not only give support to their children but may also receive so-called "upward support," which is support given by children to parents (Bordone \& de Valk, 2016). When older adults have a decreasing social network size due to retirement or losing relatives or friends, children, as a source of support, constitute an important part of the social network. For older migrants who are often not socially embedded within the larger society in the country of migration (Ciobanu et al., 2017), children may play an even more important role in later life. Theoretical assumptions pinpoint that children are a source of support and companionship to their parents, which is crucial for lower levels of loneliness (de Jong Gierveld et al., 2018). In parallel, earlier research shows that having high quality relationships with children is related to lower loneliness among Turkish migrants in Germany (Fokkema \& Naderi, 2013). However, in explaining the relatively high levels of loneliness among Turkish migrants, the role of parent-child relationships also seems somewhat ambivalent.

One explanation for this might be that social relationships are not always uniformly positive and may also encompass frictions (Parrott \& Bengtson, 1999). With regards to family relationships that are not as voluntary as compared to friendships, conflict may not result in termination of the relationship (Offer \& Fischer, 2018b), especially in the Turkish culture (Tezcan, 2018). In the context of migration, older adults might experience conflicts with their children when they perceive their children as adapting to the norms of the country of immigration (Baykara-Krumme \& Fokkema, 2019; Tezcan, 2018). In qualitative research, older Turkish migrants in Germany reported disappointment in their relationships with their children (Tezcan, 2018, 2019). For instance, "during the interviews, [older Turkish migrants] often characterised their children as disrespectful, Germanised, and assimilated" (Tezcan, 2018, p. 88). In addition, disagreements may also arise due to expectations of transmitting cultural and familial values to grandchildren, such as older migrants saying their "children [are] contributing to the high level of acculturation of grandchildren" (Tezcan, 2019, p. 12). Thus, unfulfilled expectations may be a source of conflict in parentchild relationships (Nauck, 2005), making parents feel neglected, disrespected, or disappointed and such conflicts can increase loneliness (Albert, 2021).

Against this background, the present study investigates the role of parent-child relationships in feelings of loneliness among older Turkish migrants living in Germany. Turkish migrants in Germany exemplify an ageing migrant population in Europe who feel lonely and who experience both positive and negative aspects in the relationship with their children. Such negative aspects are assumed to be related to intergenerational differences in cultural norms of the country of immigration and emigration. In this study, the second generation (i.e., born in the country of migration and both parents born in Turkey) are the children of older Turkish migrants who grew up and were socialised in Germany. Expectations with regards to interdependence and obligations in family relationships tend to be higher in the Turkish culture as compared to Germany (Baykara-Krumme \& Fokkema, 2019; Kagitcibasi \& Ataca, 2005). This may lead to friction in the parent-child relationship (Baykara-Krumme, 2010). To investigate possible conflict, we focus on filial expectations to better understand whether the expectations of parents regarding their children can explain loneliness. Whereas previous studies often compared several aspects of social relationships between Turkish migrants and native populations to explain loneliness (see van Tilburg \& Fokkema, 2020), this article contributes to the literature by focusing on the role of parent-child relationships and possible intergenerational conflict among older migrants.

In Germany, Turkish migrants represent the largest migrant population, and there is an ageing population of first-generation Turkish migrants (i.e., born in Turkey and both parents born in Turkey; Steinbach, 2018). Labour migration from Turkey to Germany started in the 1960s within the guestworker framework and continued later for family reunification purposes (Abadan-Unat \& Bilecen, 2020). Those labour migrants and their families, who arrived in the 1960s, are ageing and they are the focus of our article. Turkish migrants in Europe have worse health, SES, and housing characteristics as compared to their peers in Turkey who did not migrate (Baykara-Krumme \& Platt, 2018). In addition, older migrants have an early onset of health issues, for which they often retire at a relatively younger age (Reinprecht, 2006, as cited in Palmberger, 2019, p. 78). Using the first wave of the Generations and Gender Survey (GGS) collected among Turkish migrants in Germany in 2006, the following research question is addressed: What are the roles of parent-child relationships and filial expectations in explaining loneliness among older Turkish migrants? 


\section{Theoretical Background}

\subsection{Intergenerational Relationships and Migration}

Intergenerational relationships in migrant families can be explained by two paradoxical theories. First, according to the intergenerational solidarity thesis, family relationships become strengthened through international migration (Baykara-Krumme, 2010; Steinbach, 2013). The idea of strengthened relationships between migrant parents and their children is usually attributed to being a minority in the country of immigration, having poorer health and SES, as well as experiences of discrimination and social exclusion (Baykara-Krumme \& Fokkema, 2019). This may make migrants more focused on their immediate family as sources of support and companionship as opposed to broader social integration in the country of migration. When adult children adhere to the cultural norms of the parents, a harmonious parent-child relationship is maintained (Kwak, 2003). Second, the intergenerational conflict thesis proposes that conflict and tensions arise in migrant families. When adult children are more integrated into the country of immigration and adopt different cultural norms than their parents, conflicts and a lower contact frequency with parents might arise (Kalmijn, 2019). As described before, such tensions could make parents feel disappointed or neglected.

In research looking at both solidarity and conflict in parent-child relationships of older Turkish migrants in Germany, findings show that parent-child relationships are characterised by high solidarity and conflict at the same time (Baykara-Krumme, 2010). In sum, while both solidarity and conflict may be integral aspects in migrant families (Parrott \& Bengtson, 1999), their role in explaining loneliness among Turkish migrants might be ambivalent. Based on the intergenerational solidarity and conflict theses, we expect that solidarity and conflict in parent-child relationships influence loneliness in different ways.

\subsection{Solidarity in Parent-Child Relationships}

First, we expect that higher solidarity is related to lower loneliness. Several aspects of the relationship may foster solidarity in parent-child relationships, including aspects such as spatial proximity, contact frequency, emotional closeness, and support (Baykara-Krumme \& Fokkema, 2019; Bengtson \& Roberts, 1991). Such aspects of solidarity are also interrelated, such as higher proximity positively influencing contact frequency (Bengtson \& Roberts, 1991). Quantitative studies show high solidarity in parent-child relationships among older Turkish migrants. For instance, Turkish migrants have high relationship satisfaction and high contact frequency with their children, as well as high levels of social support exchanges within the family (Steinbach, 2013). In addition, older Turkish migrants report higher levels of co-residing with children and of emotional closeness with their children, and fewer conflicts as compared to older native Germans (Steinbach, 2018).

These findings may reflect the Turkish culture in which values such as relatedness, obligations, and interdependence with regards to family relationships are important (Kagitcibasi et al., 2010). Higher obligations for social relationships and preferences for interdependence can also be described as reflecting a collectivistic culture. This is found at the opposite spectrum of individualism, which is observed in Northwestern Europe (Baykara-Krumme \& Fokkema, 2019; Kagitcibasi \& Ataca, 2005). For instance, older Turkish adults living in the Netherlands prefer to have weekly visits from nonresiding children (de Valk \& Schans, 2008). Thus, a high contact frequency with children may fulfil the expectation of interdependency that Turkish parents have, which could lower loneliness.

Next to contact frequency, having social relationships characterised by high satisfaction is an important aspect of solidarity. Higher satisfaction with social relationships was protective against loneliness for older migrants (ten Kate et al., 2020). In research among native populations, satisfaction with social relationships was found to be a more important predictor of loneliness than contact frequency (Nicolaisen \& Thorsen, 2017). In contrast, a higher number of social interactions with someone may facilitate more opportunities for giving support to each other (Steinbach, 2013), which subsequently fosters solidarity. Having supportive social relationships that provide affection, aid, and services is related to lower levels of loneliness among Turkish migrants (Fokkema \& Naderi, 2013). Emotional support is particularly important in understanding loneliness, given that emotional support refers to "demonstrations of love and caring, esteem and value, encouragement, and sympathy" (Thoits, 2011, p. 146). Emotional support provides a sense of affection and of being valued by significant others (Thoits, 2011), which reduces loneliness. For Turkish migrants, receiving emotional support was indeed associated with lower levels of loneliness (Fokkema \& Naderi, 2013), but it is unknown whether emotional support from children also lowered loneliness. In sum, we expect that Turkish migrants who have high parent-child solidarity feel less lonely compared to those with low parent-child solidarity $(\mathrm{H} 1)$.

\subsection{Conflict and Filial Expectations}

Next to solidarity, conflict due to differences in intergenerational norms can arise in parent-child relationships, which may increase loneliness. For instance, being in conflict with family members increased loneliness among older Portuguese migrants in Luxembourg, which can also be described as a migrant population with a collectivistic culture living in a country with individualistic norms (Albert, 2021). We propose that filial expectations, which are expectations that parents have regarding interdependency with children and of caregiving obligations 
of children towards parents (de Valk \& Schans, 2008), can be a source of conflict and may explain why older Turkish migrants feel relatively lonelier. We refer to these caregiving obligations as filial expectations to highlight parents' perceptions and desires from their children.

In Turkish culture, caregiving is seen as a sign of appreciation and respect towards parents (Conkova \& Lindenberg, 2018; Tezcan, 2018) and a family obligation (Bilecen, 2020). For instance, older Turkish adults often agree with statements that children should take care of their parents when they are in need; and co-reside when parents are older to take care of them (de Valk \& Schans, 2008). Thus, the so-called filial expectations are high and are important for feeling appreciated by children for Turkish parents, in particular given the potential need for receiving care among older Turkish migrants.

As opposed to aspects of solidarity such as contact frequency and satisfaction describing the parent-child relationship, filial expectations show what is desired of the parent-child relationship. In the case of Turkish migrants living in Germany, the intergenerational conflict theory would assume that children do not meet the filial expectations of the parent when they adapt to the norms of the country of immigration (Baykara-Krumme \& Fokkema, 2019). In contrast, the solidarity thesis would expect that high filial expectations strengthen parentchild relationships when adult children adhere to the (cultural) expectations of the parent. Based on theoretical and empirical evidence, we expect the former situation (conflict) and not the latter (solidarity) with regards to filial expectations.

Turkish migrants living in Germany, a country depicted as having norms of independence, might experience a change in their parent-child relationships which can create friction in the relationship because of unmet filial expectations (Steinbach, 2018). While firstand second-generation Turkish migrants are found to be alike in expectations regarding family relationships (Baykara-Krumme \& Fokkema, 2019), it might be the case that the adherence to filial expectations is lower for the second generation (Tezcan, 2018). As children become more integrated into the country of immigration and adapt to different cultural norms as compared to their parents, parents may experience their children distancing themselves from them and from the larger migrant community (Kalmijn, 2019). Older migrants may find it difficult to accept these changes in the relationship with their children living in a country with norms of independence (Albert, 2021; Kalmijn, 2019). This change in the fulfilment of expectations can happen within one generation when adult children of migrants adapt to the norms of the country of immigration (de Valk \& Bordone, 2019). For instance, there might be more independence by adult children leaving their parental homes or outsourcing care for parents, which parents preferably receive from children themselves and not at nursing homes (Bilecen, 2020; Palmberger, 2019). Research shows that adult children share parental care duties among siblings, and when hands-on care is not possible, adult children also give financial support to parents or outsource support to others (Bilecen, 2020). From the parents' perspective, receiving support from third parties may be disappointing, given that this is not their preferred choice of personal care as desired in the Turkish culture (Palmberger, 2019). Not receiving the care and attention from children as desired made some Turkish parents describe their children as "disrespectful," which created conflict and sometimes even led parents to refuse to talk to their children temporarily (Tezcan, 2018).

In sum, unmet filial expectations might increase loneliness. Some scholars argue that in collectivistic cultures with higher interdependence standards, such as in the Turkish culture, feelings of loneliness may arise, given that higher expectations on social relationships are more difficult to fulfil than in individualistic cultures with lower interdependence standards (Lykes \& Kemmelmeier, 2014). Hence, loneliness can even be described as a response to not meeting cultural expectations (Lykes \& Kemmelmeier, 2014), which may be the case regarding the filial expectations of Turkish migrants. Such differences between parents and children can increase conflict in the relationship, which increases loneliness (Albert, 2021). Therefore, given that having higher expectations indicates a higher risk of unfulfilled expectations, Turkish migrants who have higher filial expectations are lonelier than those with lower expectations $(\mathrm{H} 2)$.

\subsection{Unmet Expectations}

For loneliness, it is mainly the discrepancy in the fulfilment of filial expectations that explains loneliness rather than merely having high filial expectations. The fulfilment of filial expectations depends on individual circumstances, such as whether care is needed in the first place. After all, "strong feelings of family responsibility may predispose individuals to be supportive, but whether assistance actually materialises depends on the specific context of need" (Dykstra et al., 2013, pp. 33-34). Hence, when a discrepancy arises between expected support and given support, the result is a higher level of loneliness.

An important factor with regards to the fulfilment of filial expectations is intergenerational co-residence, given that it influences opportunities to receive support from children (Dykstra et al., 2013). Co-residing children may fulfil filial expectations better than nonresiding children, as the former can give hands-on care. If parents have high filial expectations and also have coresiding children, they will have fewer unmet expectations as compared to those with high expectations who do not have co-residing children, lowering loneliness. Thus, Turkish migrants who have higher filial expectations and have co-residing children are less lonely than those without co-residing children $(\mathrm{H} 2 \mathrm{a})$. 
In addition, health is a major determinant of care needs and loneliness. The need to receive care increases as health worsens and this, subsequently, could increase discrepancies between filial expectations and received filial care, thus increasing loneliness. Older migrants have, on average and at a relatively young age, relatively many health problems and a low perceived health, partly resulting from poor working conditions in low-skilled jobs (Ciobanu et al., 2017). Such health issues may indicate a relatively high need for receiving care. Moreover, income difficulties can also indicate a need for support. It is known that most Turkish migrants prefer to receive this kind of care and support from their children, rather than from the welfare state (Bilecen, 2020; Palmberger, 2019). However, when adult children, who are socialised in Germany, do not meet these needs with regards to health and income difficulties, this would lead to unmet filial expectations and, subsequently, to more loneliness. For parents who have poor health and income difficulties, having lower filial expectations would decrease loneliness given there is less of a risk of unmet filial expectations. We, therefore, expect that poor health coupled with income difficulties strengthen the association of H2: Turkish migrants who have higher filial expectations and poor health $(\mathrm{H} 2 \mathrm{~b})$ or income difficulties $(\mathrm{H} 2 \mathrm{C})$ feel lonelier than those with good health and few income difficulties.

\section{Method}

\subsection{Study Sample}

We use the first wave of the GGS Turkish-German subsample, which was collected in 2006. For the sampling, an estimation was made of Turkish respondents aged between 18 and 79 in all German municipalities who are not living in residential or nursing homes. A random selection was then made of respondents within these municipalities (Fokkema \& Naderi, 2013; GGS Online Codebook and Analysis, n.d.). Out of the 9,711 eligible respondents, a total of 4,045 completed questionnaires were collected, showing a similar response rate (41.6\%) as a survey among older Turkish migrants living in the Netherlands (i.e., $45 \%$ in the Longitudinal Aging Study Amsterdam collected in 2013-2014; see also van Tilburg \& Fokkema, 2020).

Trained interviewers visited the respondents at home and interviewed them face-to-face, using standardised questionnaires (available in the German and Turkish language) to collect data. The Turkish questionnaires were translated only forward, and not backward, which might have the risk of misinterpretation by the respondents when some questions were posed in different languages by the interviewers. Survey topics revolved around partnerships, children, household members, SES, health, loneliness, and social support exchanges. Respondent incentives were up to 10 euros and respondents were informed about the study via a letter before interview- ers visited them at home. The average length of the interview was 65 minutes (in German) and 88 minutes (in Turkish; Fokkema \& Naderi, 2013).

For our analysis, we selected respondents who (a) are first-generation Turkish migrants, determined by the country of birth of the respondent, (b) had at least one child, and (c) were aged 50 years or older. Our final sample consists of 606 respondents.

\subsection{Measurements}

\subsubsection{Loneliness}

Loneliness was measured by the six-item de Jong Gierveld scale $(\alpha=0.78)$, which was tested as psychometrically sound for older Turkish migrants (van Tilburg \& Fokkema, 2020). The scale has been used in several studies to measure loneliness among older migrants (see Fokkema \& Naderi, 2013; Klok et al., 2017; ten Kate et al., 2020). For three items, respondents indicated whether they have enough or many relationships that provide them with a sense of closeness, support when needed, or people whom they trust. For the other three items, respondents indicated whether they experience feelings of emptiness, feeling rejected, or miss having people around. Respondents could answer yes, more or less, and no. For the first three items, the categories more or less and no count as lonely, and for the latter three items, yes and more or less count as lonely (de Jong Gierveld \& van Tilburg, 2017).

\subsubsection{Parent-Child Relationships}

For aspects that are likely to reflect solidarity in parentchild relationships, we use four indicators.

Co-residing was measured by the number of adult coresiding children aged 18 and above in the household. Respondents could list for each household member who this person was (e.g., child), and their age. Based on this information, we counted the number of co-residing children aged 18 and over.

Contact frequency is measured by the number of children living outside the household with whom the respondent meets at least weekly. Respondents could list for each non-residing child how often they meet. Hence, we counted the number of non-residing children that the respondent meets daily, several times a week, or weekly.

Relationship satisfaction was measured by the question of how satisfied the respondent is with the relationship with each child living outside the household on a scale from 0 (not at all satisfied) to 10 (completely satisfied). Information on satisfaction with the relationship with co-residing children was not available. Scores below 7 are considered to be low-satisfying relationships and scores of 7 and higher are considered to be highsatisfying relationships (Fokkema \& Naderi, 2013). Based on this, two variables were constructed, indicating (a) the number of low-satisfying relationships with non-resident 
children and (b) the number of high-satisfying relationships with non-resident children.

For emotionally supportive relationships with children, respondents could list up to five people with whom they talked about personal feelings and problems in the last 12 months. Next, they indicated for each person the type of relationship (e.g., their partner, child, or friend). From this, the number of emotionally supportive relationships with children was derived, which could vary from 0 to 5 .

\subsubsection{Filial Expectations}

For filial expectations, a scale $(\alpha=0.79)$ was constructed that shows the average regarding five statements: (a) children should take responsibility for parental care if parents are in need; (b) children should adjust their working lives to the needs of their parents; (c) when parents are in need, daughters should take caring responsibility; (d) children should provide financial help if parents have financial difficulty; and (e) children should live with parents when parents can no longer look after themselves. Respondents could indicate from 1 (disagree) to 5 (high agreement) their agreement with each of the statements. We decided on these five items as they measure parents' perceptions of children's support obligations towards their parents.

\subsubsection{Health and Income Difficulties}

Health is measured by two variables. First, perceived health is measured by the question: How is your health in general? Answer possibilities were 0 (very bad), 1 (bad), 2 (fair), 3 (good), or 4 (very good). Low perceived health is related to lower levels of loneliness among older migrants and has an effect on loneliness above and beyond merely objective health indicators (ten Kate et al., 2020; Visser \& El Fakiri, 2016). Second, objective health is measured by whether respondents have a longstanding illness or a chronic disease, or a health-related limitation or disability. Respondents who have answered no to both of these questions were considered to have no disease or disability. Income difficulties show the perceived difficulty of making ends meet on a scale from 0 (not difficult at all) to 5 (very difficult).

\subsubsection{Controls}

We control for the possible influence of the following variables on loneliness: partner, emotional support, and socio-demographics.

We include whether respondents have a partner and are satisfied with the relationship. Respondents could rate their satisfaction with the relationship with their partner on a scale from 0 (not satisfied) to 10 (very satisfied). Relationships scoring below 7 are considered as low-satisfying relationships and those scoring 7 and higher as high-satisfying relationships. Having a partner and having a satisfactory relationship with the partner is related to less loneliness as it fulfils the need for having a close bond (ten Kate et al., 2020).

Based on the types of social contacts besides the children from whom the respondent received emotional support, we also include the number of people besides children from whom respondents received emotional support. In addition, respondents could list up to five people who talked about their personal feelings or problems to the respondent. Based on this, the number of people to whom the respondent gave emotional support was constructed. Giving emotional support to others gives a sense of mattering in life and feeling appreciated by significant others (Thoits, 2011), which may reduce loneliness.

For socio-demographics, we include age, gender, and whether respondents are retired or homemakers, the latter being described as looking after the home or family.

\subsection{Analyses}

In complete case stepwise linear multivariate regression analyses we first add the control variables and aspects of parent-child relationships to test $\mathrm{H} 1$. We then add filial expectations to test $\mathrm{H} 2$. Next, health, income difficulties, and interaction terms were added to test hypotheses $2 \mathrm{a}, 2 \mathrm{~b}$, and $2 \mathrm{c}$. To avoid multicollinearity, the continuous variables were first centred before constructing the interactions. For $\mathrm{H} 2 \mathrm{a}$, an interaction term was made between having adult co-residing children and filial expectations; for $\mathrm{H} 2 \mathrm{~b}$, between perceived health and filial expectations, and between objective health and filial expectations; and for H2c, between income difficulties and filial expectations. With regards to missing data, the percentage of missing values was highest on the variables of loneliness (6.8\%) and filial expectations (5.8\%) and was below $5 \%$ on all other variables. Listwise deletion was used for missing data.

\section{Results}

\subsection{Descriptives}

Table 1 shows the description of the sample, indicating that, on average, respondents have a moderate level of loneliness, with $45.1 \%$ having some feelings of loneliness, and $11.9 \%$ experiencing severe loneliness. With regards to parent-child relationships, about half of the respondents $(52.3 \%)$ have at least one adult co-residing child. Of the respondents who have non-residing children $(59.9 \%$ of the sample), $37.1 \%$ have weekly contact and $56.9 \%$ are satisfied with the relationship with at least one non-residing child. In addition, the average of 0.17 on receiving emotional support from children shows that most respondents do not list their children as emotionally supportive ties. The mean value on filial expectations of 3.29 on a scale from 1 to 5 shows an agreement that children have a responsibility to take care of parents 
Table 1. Descriptives of older Turkish migrants living in Germany $(n=606)$.

\begin{tabular}{|c|c|c|c|c|}
\hline Variable & $\%$ & $n$ & Mean (se) & $\begin{array}{l}\text { 25th, 50th, and } \\
\text { 75th percentile }\end{array}$ \\
\hline Loneliness & & & $1.91(0.08)$ & $0,1,3$ \\
\hline Not lonely (0-1) & 48.2 & 292 & & \\
\hline Moderately lonely (2-4) & 33.2 & 201 & & \\
\hline Severely lonely (5-6) & 11.9 & 72 & & \\
\hline$n$ adult co-residing children & & & $0.95(0.05)$ & $0,1,1$ \\
\hline 0 & 48.8 & 296 & & \\
\hline 1 & 32.3 & 196 & & \\
\hline $2-3$ & 14.1 & 85 & & \\
\hline $4-8$ & 4.9 & 29 & & \\
\hline$n$ non-residing children with weekly contact & & & $0.67(0.04)$ & $0,0,1$ \\
\hline 0 & 62.4 & 378 & & \\
\hline 1 & 17.7 & 107 & & \\
\hline $2-3$ & 17.4 & 109 & & \\
\hline $4-6$ & 2.0 & 12 & & \\
\hline$n$ low-satisfying relationships with children & & & $0.20(0.03)$ & $0,0,0$ \\
\hline 0 & 88.1 & 534 & & \\
\hline 1 & 6.9 & 42 & & \\
\hline $2-4$ & 4.9 & 30 & & \\
\hline$n$ high-satisfying relationships with children & & & $1.49(0.07)$ & $0,1,3$ \\
\hline 0 & 43.2 & 262 & & \\
\hline 1 & 12.0 & 73 & & \\
\hline $2-3$ & 32.5 & 197 & & \\
\hline $4-10$ & 12.4 & 75 & & \\
\hline$n$ children giving the respondent emotional support & & & $0.17(0.02)$ & $0,0,0$ \\
\hline 0 & 88.4 & 536 & & \\
\hline 1 & 8.1 & 49 & & \\
\hline $2-4$ & 3.5 & 20 & & \\
\hline Filial expectations & & & $3.29(0.04)$ & $2.4,3,4.2$ \\
\hline Perceived health & & & $2.29(0.04)$ & $2,2,3$ \\
\hline Having a disease, illness and/or disability & 42.4 & 257 & & \\
\hline Income difficulties & & & $2.94(0.05)$ & $2,3,4$ \\
\hline Having a partner & 81.2 & 492 & & \\
\hline High satisfaction with partner & 76.2 & 462 & & \\
\hline$n$ ties besides children giving the respondent emotional support & & & $0.32(0.03)$ & $0,0,1$ \\
\hline$n$ ties respondent has given emotional support to & & & $0.42(0.04)$ & $0,0,0.25$ \\
\hline Women & 47.9 & 290 & & \\
\hline Age & & & $58.74(0.25)$ & $54,58,63$ \\
\hline Retired & 35.1 & 213 & & \\
\hline Homemaker & 21.9 & 133 & & \\
\hline
\end{tabular}

who are in need. For bivariate associations, we refer to Tables S1 and S2 of the Supplementary Material.

\subsection{Hypotheses Testing}

In Table 2, the results of the linear regression model are displayed. For $\mathrm{H} 1$, which described that solidarity in parent-child relationships is related to lower loneliness, there is partial support given that two out of the four aspects of solidarity are related to loneliness. First, having adult co-residing children is related to lower loneliness. Second, having low-satisfying relationships with non-residing children increases loneliness. Having high-satisfying relationships with non-residing children, weekly contact frequency, or receiving emotional support from children were not related to lower loneliness, suggesting that discrepancies (e.g., not being satisfied) predict loneliness, but fulfilled expectations do not 
Table 2. Linear regression models for loneliness $(n=524)$.

\begin{tabular}{|c|c|c|c|}
\hline \multirow[t]{2}{*}{ Variable } & Model 1 & Model 2 & Model 3 \\
\hline & $\mathrm{B}^{\mathrm{a}}(\mathrm{se})$ & $\mathrm{B}^{\mathrm{a}}(\mathrm{se})$ & $\mathrm{B}^{\mathrm{a}}(\mathrm{se})$ \\
\hline Constant & $2.92(0.46)$ & $2.69(0.51)$ & $2.82(0.61)$ \\
\hline Perceived health & $-0.26(0.10) * *$ & $-0.28(0.10)^{* *}$ & $-0.27(0.10)^{* *}$ \\
\hline Having a disease or illness ( $1=$ yes) & $0.29(0.19)$ & $0.27(0.19)$ & $0.28(0.19)$ \\
\hline Income difficulties & $0.18(0.07)^{* *}$ & $0.17(0.07)^{* *}$ & $0.17(0.07)^{* *}$ \\
\hline Having a partner ( $1=$ yes $)$ & $0.48(0.35)$ & $0.51(0.356)$ & $0.45(0.356)$ \\
\hline Satisfaction with partner high ( 1 = yes) & $-1.20(0.34) * * *$ & $-1.22(0.34)^{* * *}$ & $-1.21(0.34)^{* * *}$ \\
\hline R. receiving emotional support besides children & $-0.16(0.17)$ & $-0.16(0.18)$ & $-0.15(0.18)$ \\
\hline R. given emotional support to others & $-0.22(0.13)$ & $-0.21(0.13)$ & $-0.19(0.13)$ \\
\hline Age $^{b}$ & $-0.01(0.02)$ & $-0.01(0.02)$ & $-0.01(0.02)$ \\
\hline Gender (1 = women) & $0.02(0.20)$ & $0.01(0.20)$ & $0.01(0.20)$ \\
\hline Being retired $(1=$ yes $)$ & $-0.20(0.21)$ & $-0.21(0.20)$ & $-0.19(0.20)$ \\
\hline Being a homemaker (1 = yes) & $-0.37(0.24)$ & $-0.37(0.24)$ & $-0.40(0.24)$ \\
\hline H1. N. adult co-residing child(ren) & $-0.20(0.07)^{* *}$ & $-0.19(0.07)^{* *}$ & $-0.19(0.07)^{* *}$ \\
\hline H1. N. weekly contact child(ren) & $-0.07(0.09)$ & $-0.07(0.09)$ & $-0.06(0.09)$ \\
\hline H1. N. low satisfaction with child(ren) ${ }^{c}$ & $0.39(0.13)^{* *}$ & $0.39(0.13)^{* *}$ & $0.38(0.13)^{* *}$ \\
\hline H1. N. high satisfaction with child(ren $)^{c}$ & $-0.10(0.07)$ & $-0.10(0.07)$ & $-0.10(0.07)$ \\
\hline H1. N. child(ren) giving emotional support & $0.07(0.18)$ & $0.06(0.18)$ & $-0.01(0.18)$ \\
\hline H2. Filial expectations & & $0.09(0.08)$ & $0.05(0.12)$ \\
\hline H2a. Adult co-residing child $\times$ filial expectations ${ }^{d}$ & & & $-0.05(0.06)$ \\
\hline H2b. Perceived health $\times$ filial expectations ${ }^{d}$ & & & $0.25(0.10)^{* *}$ \\
\hline $\mathrm{H} 2 \mathrm{~b}$. Disease $\times$ filial expectations ${ }^{d}$ & & & $0.19(0.19)$ \\
\hline H2c. Income difficulties $\times$ filial expectations ${ }^{d}$ & & & $-0.03(0.07)$ \\
\hline$R^{2}$ & 0.08 & 0.18 & 0.23 \\
\hline$R^{2}$ adjusted & 0.07 & 0.16 & 0.20 \\
\hline F-change model & $9.38 * * *$ & 1.29 & 2.04 \\
\hline
\end{tabular}

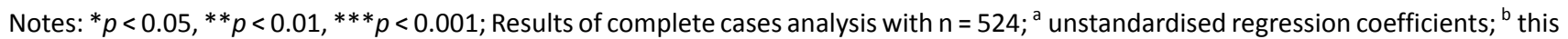
variable is centred for a better interpretation of respondents who score 0 on age (mean age $=58.74$ ); ${ }^{c}$ satisfaction with non-residing children; ${ }^{d}$ continuous variables were centred before making the interaction term to avoid multicollinearity (this means that the slope shows the effect of scoring above average on the variables); mean values are perceived health $=2.29$, income difficulties $=2.94$, filial expectations $=3.29$.

(e.g., weekly contact, satisfying relationships, receiving emotional support).

$\mathrm{H} 2$, which stated that older Turkish migrants who have higher filial expectations are lonelier, is not supported. The small insignificant positive effect of filial expectations on loneliness had a high standard error, showing variation in the association between filial expectations and loneliness. No support was found for $\mathrm{H} 2 \mathrm{a}$, $\mathrm{H} 2 \mathrm{~b}$, and $\mathrm{H} 2 \mathrm{c}$, focusing on the interaction between coresiding children, health, income difficulties with filial expectations on loneliness (see model 3 ). Interaction effects of observed values for $\mathrm{H} 2 \mathrm{~b}$ with regards to perceived health are shown in Figure 1 and $\mathrm{H} 2 \mathrm{a}$ until $\mathrm{H} 2 \mathrm{c}$ are depicted in Supplementary Material (Figures S1 to $\mathrm{S} 3$ ). Contrary to $\mathrm{H} 2 \mathrm{~b}$, the positive and significant slope of perceived health multiplied by filial expectations shows that respondents who have an above-average perceived health and above average filial expectations, have higher levels of loneliness. Respondents who have belowaverage perceived health are less lonely when they have higher filial expectations. This is depicted in Figure 1, showing that respondents who have fair or good perceived health and higher filial expectations have a higher level of loneliness. Moreover, the scores on loneliness are around a value of 2 (moderate loneliness) when respondents have higher filial expectations, regardless of their health status.

\section{Discussion and Conclusion}

This article focused on the role of parent-child relationships in explaining loneliness among Turkish migrants 


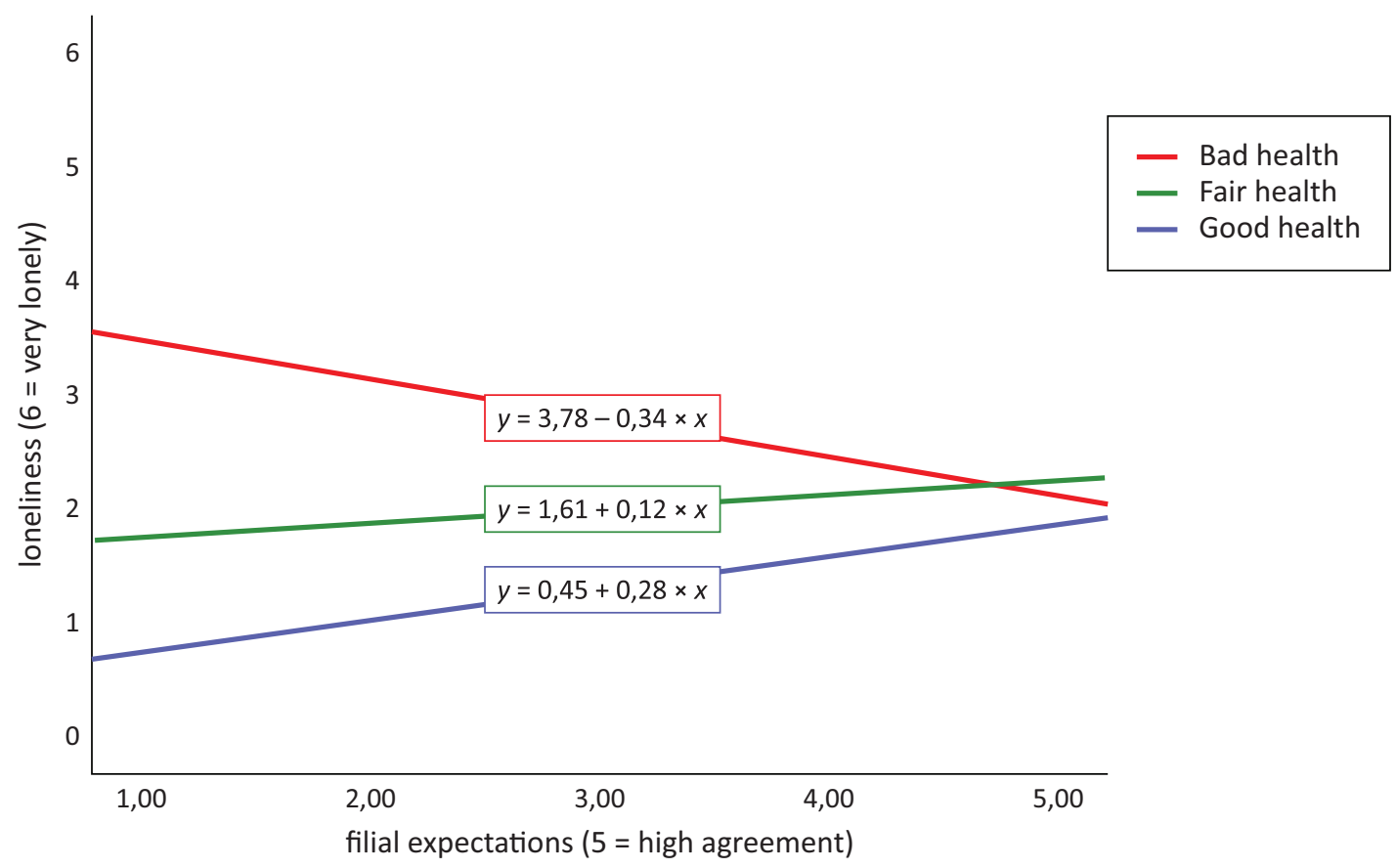

Figure 1. Interaction of observed values between loneliness and filial expectations for categories of perceived health.

in Germany. In Germany, as well as in other European countries, migrants age alongside native populations. This not only has demographical but also societal consequences, given the stronger feelings of loneliness among older migrants. Older Turkish migrants are an example of an older migrant population who have stronger feelings of loneliness, which can be seen as the opposite of social inclusion and embeddedness. Higher loneliness has several negative outcomes on both mental and physical health, highlighting the importance of a better understanding of high loneliness. While some research shows a high family cohesion for Turkish migrant families that could prevent loneliness, there is also ambivalence in parent-child relationships. Such ambivalence may be related to the social integration of adult children (i.e., the second generation) who adapt to the norms of the country of immigration, which could disappoint parents who have the norms of the country of emigration (Kalmijn, 2019).

We contribute to the literature by focusing on several aspects of parent-child relationships that foster solidarity, and on the role of filial expectations as a potential source of conflict in parent-child relationships that may increase loneliness. Certain aspects of solidarity, such as having co-residing children, are related to lower loneliness, but high satisfaction with children, contact frequency, or receiving emotional support from children are not. Our findings thus challenge previous studies assuming that "good" relationships always reduce loneliness, given that there was no association found between several aspects of solidarity in parent-child relationships and loneliness. As for the conflict thesis, qualitative studies showed that some Turkish migrants have conflicts in the relationship with their children, due to filial and cultural expectations (Tezcan, 2018, 2019), which may increase feelings of loneliness. However, we found that filial expectations had no independent effect on loneliness, suggesting that expectations themselves are not necessarily a risk for loneliness.

Moreover, we found that Turkish migrants with fair or good perceived health and higher filial expectations have higher levels of loneliness. This suggests that older Turkish migrants with worse perceived health may get satisfactory support and care from their children, and hence have less often unfulfilled filial expectations compared to those with better perceived health. This finding indicates that when parents have worse health, children may perceive their parents' needs, and are ready to care for them. Perhaps for parents with worse health, the parent-child relationships are more akin to solidarity than to conflict. Interestingly, older Turkish migrants with worse health feel lonelier when they do not have high expectations from children. This suggests that Turkish migrant parents with lower expectations may have more friction in their relationships with their children. It might also be the case that older migrants with low filial expectations experience a deviation from what is expected in the Turkish culture, which could increase loneliness. These findings are crucial to understanding the effects of health needs concerning filial expectations explaining loneliness among older migrants living in Europe who migrated from a country with a collectivistic culture.

Although our study has its contributions, there are some limitations. First, filial expectations were measured by statements describing what is expected of children in general. Therefore, it is unknown whether older Turkish migrants have the same filial expectations when it comes to their own children and situation. For instance, some 
older migrants describe that they do not want to be a burden to their children and may accept formal care if needed (Conkova \& Lindenberg, 2018; Palmberger, 2019). Second, receiving instrumental support or financial support from children was not included in the present study. In the data, only a handful of respondents indicated that they received help with personal care or financial support from their children. We, therefore, could not include receiving instrumental and financial support in our analyses, but these types of support may have an impact on loneliness. Last, the data did not include information on migration characteristics or life course events, such as feelings of belonging to Germany, the motivation for migrating, or changes in health or SES over the years. While the years of residing in Germany and the age of migration were not related to loneliness (see Supplementary Material, Table S3), migration characteristics and life course events may play a role in loneliness.

For future studies, we have three recommendations. First, the role of receiving instrumental and financial support from children, and the possible outsourcing of caregiving should be further considered. Care responsibilities can also be fulfilled by a third party, which is not preferred by older Turkish migrants (Bilecen, 2020; Palmberger, 2019). This may still be a viable option for care that can lower unfulfilled filial expectations, which, in turn, might decrease loneliness. Here, it should also be considered how the health and SES of older Turkish migrants influence the extent to which they receive care from their children, and subsequently, loneliness. Second, the role of gender in parent-child relationships needs closer examination, in particular in the context of the Turkish culture placing more caregiving responsibilities on daughters than on sons (Conkova \& Lindenberg, 2018; Kagitcibasi et al., 2010). Some gender differences were also found in the GGS data (see Supplementary Material, Table S4), but this needs further examination. For instance, research on relationships between daughters, sons, fathers, and mothers with a Turkish migration background shows distinct patterns, such as daughters having both more conflicting and harmonious relationships with their parents as compared to sons (Baykara-Krumme, 2010). Third, while our study focused on feelings of overall loneliness, loneliness can also be theorised and analysed as a bidimensional concept (Pinquart \& Sörensen, 2001; van Baarsen et al., 2001). Social loneliness (i.e., feelings of embeddedness to a larger social network) and emotional loneliness (i.e., lack of strong emotional bonds) may be related differently to parent-child relationships. For instance, whereas lack of engagement in social activities with children could explain social loneliness, low relationship satisfaction and emotional support could explain emotional loneliness. While we did find some differences according to the type of loneliness (such as the interaction between filial expectations and perceived health predicting social loneliness but not emotional loneliness; see
Supplementary Material, Table S5), future studies may look into this issue more in-depth.

In sum, parent-child relationships play an ambivalent role in explaining loneliness among older Turkish migrants in Germany. Several aspects of solidarity in parent-child relationships such as receiving emotional support are not associated with loneliness. For older Turkish migrants who have relatively high filial expectations, this may explain both higher and lower levels of loneliness. These findings indicate that especially healthy older Turkish migrants may have unfulfilled expectations regarding parent-child relationships, which adds to their loneliness, while parents with poor health experience solidarity, which lowers their loneliness. This paints a picture of both intergenerational solidarity and conflict: Turkish migrants maintain close relationships with their adult children, and expectations on caregiving responsibilities from children may be fulfilled for parents who have worse perceived health, but not for those with good perceived health. This suggests that in such situations, children also perceive the needs of their parents and fulfil the expected caregiving role. The interplay of ageing, migration, and filial expectations in what constitutes a satisfactory social relationship and social support from children-and its impact on loneliness-should be considered in future research and in policy interventions aiming to reduce loneliness among older migrants.

\section{Acknowledgments}

We would like to thank the editor and anonymous reviewers for their helpful comments on earlier drafts. We also thank our colleagues of the FLAG research group at the University of Groningen Sociology Department for their comments and suggestions.

\section{Conflict of Interests}

The authors declare no conflict of interests.

\section{Supplementary Material}

Supplementary material for this article is available online in the format provided by the author (unedited).

\section{References}

Abadan-Unat, N., \& Bilecen, B. (2020). The TurkeyGermany migration corridor. In T. Bastia \& R. Skeldon (Eds.), Routledge handbook of migration and development (pp. 458-463). Routledge. http://dx.doi.org/ 10.4324/9781315276908-42

Albert, I. (2021). Perceived loneliness and the role of cultural and intergenerational belonging: The case of Portuguese first-generation immigrants in Luxembourg. European Journal of Ageing, 66, 299-310. https://doi.org/10.1007/s10433-021-00617-7

Baykara-Krumme, H. (2010). Explaining family cohesion 
in an immigration context: Patterns of intergenerational relationship quality among young adult Turks and their parents (Working Paper No. 20). Panel Analysis of Intimate Relationships and Family Dynamics. https://www.ssoar.info/ssoar/handle/ document/36947

Baykara-Krumme, H., \& Fokkema, T. (2019). The impact of migration on intergenerational solidarity types. Journal of Ethnic and Migration Studies, 45(10), 1707-1727. https://doi.org/10.1080/1369183X. 2018.1485203

Baykara-Krumme, H., \& Platt, L. (2018). Life satisfaction of migrants, stayers and returnees: Reaping the fruits of migration in old age? Ageing and Society, 38, 721-745. http://dx.doi.org/10.1017/ S0144686X16001227

Bengtson, V. L., \& Roberts, R. E. L. (1991). Intergenerational solidarity in aging families: An example of formal theory construction. Journal of Marriage and the Family, 53(4), 856-870. https://doi.org/10.2307/ 352993

Bilecen, B. (2020). Asymmetries in transnational social protection: Perspectives of migrants and nonmigrants. The Annals of the American Academy of Political and Social Science, 689(1), 168-191. https://doi. org/10.1177/0002716220922521

Bordone, V., \& de Valk, H. A. G. (2016). Intergenerational support among migrant families in Europe. European Journal of Ageing, 13(3), 259-270. https://doi.org/ 10.1007/s10433-016-0363-6

Ciobanu, R. O., Fokkema, T., \& Nedelcu, M. (2017). Ageing as a migrant: Vulnerabilities, agency and policy implications. Journal of Ethnic and Migration Studies, 43(2), 164-181. https://doi.org/10.1080/1369183X. 2016.1238903

Conkova, N., \& Lindenberg, J. (2018). Gezondheid en welbevinden van oudere migranten in Nederland: Een narratieve literatuurstudie [Health and wellbeing of older migrants in the Netherlands: A narrative literature study]. Tijdschrift voor Gerontologie en Geriatrie, 49, 223-231. https://doi.org/10.1007/s12439018-0268-2

de Jong Gierveld, J., \& van Tilburg, T. G. (2006). A 6item scale for overall, emotional, and social loneliness: Confirmatory tests on survey data. Research on Aging, 28, 582-598. https://doi.org/10.1177/ 0164027506289723

de Jong Gierveld, J., \& van Tilburg, T. G. (2017, June 15). Updated manual of the Loneliness Scale 1999. VU University. https://home.fsw.vu.nl/tg.van. tilburg/manual_loneliness_scale_1999.html

de Jong Gierveld, J., van Tilburg, T. G., \& Dykstra, P. A. (2018). New ways of theorizing and conducting research in the field of loneliness and social isolation. In A. L. Vangelisti \& D. Perlman (Eds.), The Cambridge handbook of personal relationships (pp. 391-404). Cambridge University Press. https://doi.org/ 10.1017/9781316417867.031 de Valk, H. A. G., \& Bordone, V. (2019). Co-residence of adult children with their parents: Differences by migration background explored and explained. Journal of Ethnic and Migration Studies, 45(10), 1790-1812. https://doi.org/10.1080/1369183X. 2018.1485207

de Valk, H. A. G., \& Schans, D. (2008). "They ought to do this for their parents": Perceptions of filial obligations among immigrant and Dutch older people. Ageing and Society, 28(1), 49-66. https://doi.org/ $10.1017 / \mathrm{s} 0144686 \times 07006307$

Dykstra, P. A., van Den Broek, T., , Muresan, C., Haragus, M., Haragus, P.-T., Abramowska-Kmon, A., \& Kotowska, I. E. (2013). State-of-the-art report. Intergenerational linkages in families. Families and Societies, 1, 1-47. http://www.familiesandsocieties. eu/wp-content/uploads/2014/12/WP01Dykstra Etal2013.pdf

Fokkema, T., \& Naderi, R. (2013). Differences in latelife loneliness: A comparison between Turkish and native-born older adults in Germany. European Journal of Ageing, 10(4), 289-300. https://doi.org/ 10.1007/s10433-013-0267-7

GGS Online Codebook \& Analysis. (n.d.). Generations and Gender Survey: Germany-Turkish subsample wave 1 \& wave 2 [Data set]. https://ggpsurvey. ined.fr/webview/index.jsp?study=http\%3A\%2F\% 2Fggpsurvey.ined.fr\%3A80\%2Fobj\%2FfStudy\% 2FGGS.W1.W2.14.51\&node $=0$ \& mode $=$ download \& $\mathrm{v}=2$ \&analysismode=table \&top=yes\&language $=$ en

Holt-Lunstad, J., Smith, T. B., Baker, M., Harris, T., \& Stephenson, D. (2015). Loneliness and social isolation as risk factors for mortality. Perspectives on Psychological Science, 10(2), 227-237. https://doi.org/ 10.1177/1745691614568352

Kagitcibasi, C., \& Ataca, B. (2005). Value of children and family change: A three-decade portrait from Turkey. Applied Psychology, 54(3), 317-337. https://doi.org/ 10.1111/j.1464-0597.2005.00213.x

Kagitcibasi, C., Ataca, B., \& Diri, A. (2010). Intergenerational relationships in the family: Ethnic, socioeconomic, and country variations in Germany, Israel, Palestine, and Turkey. Journal of Cross-Cultural Psychology, 41(5), 652-670. https://doi.org/10.1177/ 0022022110372193

Kalmijn, M. (2019). Contact and conflict between adult children and their parents in immigrant families: Is integration problematic for family relationships? Journal of Ethnic and Migration Studies, 45(9), 1419-1438. https://doi.org/10.1080/1369183X. 2018.1522245

Klok, J., van Tilburg, T. G., Suanet, B., Fokkema, T., \& Huisman, M. (2017). National and transnational belonging among Turkish and Moroccan older migrants in the Netherlands: Protective against loneliness? European Journal of Ageing, 14(4), 341-351. https://doi. org/10.1007/s10433-017-0420-9

Kwak, K. (2003). Adolescents and their parents: A 
review of intergenerational family relations for immigrant and non-immigrant families. Human Development, 46 (2/3), 115-136. https://doi.org/10.1159/ 000068581

Lykes, V. A., \& Kemmelmeier, M. (2014). What predicts loneliness? Cultural difference between individualistic and collectivistic societies in Europe. Journal of Cross-Cultural Psychology, 45(3), 468-490. https:// doi.org/10.1177/0022022113509881

Nauck, B. (2005). Intergenerational relations in Turkish families in Germany. In J. Pflegerl \& S. Trnka (Eds.), Migration and the family in the European Union (pp. 99-128). Austrian Institute for Family Studies. https://www.researchgate.net/publication/ 260479342_Intergenerational_Relations_in_ Turkish_Families_in_Germany

Nicolaisen, M., \& Thorsen, K. (2017). What are friends for? Friendships and loneliness over the lifespanFrom 18 to 79 years. International Journal of Aging and Human Development, 84(2), 126-158. https:// doi.org/10.1177/0091415016655166

Offer, S., \& Fischer, C. S. (2018a). Calling on kin: The place of parents and adult children in egocentric networks. In D. F. Alwin, D. H. Felmlee, \& D. A. Kreager (Eds.), Social networks and the life course: Integrating the development of human lives and social relational networks (pp. 117-138). Springer. https:// doi.org/10.1007/978-3-319-71544-5_6

Offer, S., \& Fischer, C. S. (2018b). Difficult people: Who is perceived to be demanding in personal networks and why are they there? American Sociological Review, 83(1), 111-142. https://doi.org/10.1177/ 0003122417737951

Palmberger, M. (2019). Relational ambivalence: Exploring the social and discursive dimensions of ambivalence-The case of Turkish aging labor migrants. International Journal of Comparative Sociology, 60(1/2), 74-90. https://doi.org/10.1177/ 0020715219832918

Parrott, T. M., \& Bengtson, V. L. (1999). The effects of earlier intergenerational affection, normative expectations, and family conflict on contemporary exchanges of help and support. Research on Aging, 21(1), 73-105. https://doi.org/10.1177/0164027599 211004
Pinquart, M., \& Sörensen, S. (2001). Influences on loneliness in older adults: A meta-analysis. Basic and Applied Social Psychology, 23(4), 245-266. https:// doi.org/10.1207/153248301753225702

Steinbach, A. (2013). Family structure and parent-child contact: A comparison of native and migrant families. Journal of Marriage and Family, 75(5), 1114-1129. https://doi.org/10.1111/jomf.12060

Steinbach, A. (2018). Older migrants in Germany. Journal of Population Ageing, 11(3), 285-306. https:// doi.org/10.1007/s12062-017-9183-5

ten Kate, R. L. F., Bilecen, B., \& Steverink, N. (2020). A closer look at loneliness: Why do first-generation migrants feel more lonely than their native Dutch counterparts? Gerontologist, 60(2), 291-301. https://doi.org/10.1093/geront/gnz192

Tezcan, T. (2018). On the move in search of health and care: Circular migration and family conflict amongst older Turkish immigrants in Germany. Journal of Aging Studies, 46, 82-92. https://doi.org/10.1016/ j.jaging.2018.07.001

Tezcan, T. (2019). First-generation circular migrants involved in the upbringing of their grandchildren: The case of Turkish immigrants in Germany. Ageing and Society, 41(1), 77-100. https://doi.org/10.1017/ S0144686X19000953

Thoits, P. A. (2011). Mechanisms linking social ties and support to physical and mental health. Journal of Health and Social Behavior, 52, 145-161. https://doi. org/10.1177/0022146510395592

van Baarsen, B., Snijders, T. A. B., Smit, J. H., \& van Duijn, M. A. J. (2001). Lonely but not alone: Emotional isolation and social isolation as two distinct dimensions of loneliness in older people. Educational and Psychological Measurement, 61(1), 119-135. https:// doi.org/10.1177/00131640121971103

van Tilburg, T. G., \& Fokkema, T. (2020). Stronger feelings of loneliness among Moroccan and Turkish older adults in the Netherlands: In search for an explanation. European Journal of Ageing, 18, 311-322. https://doi.org/10.1007/s10433-020-00562-x

Visser, M. A., \& El Fakiri, F. (2016). The prevalence and impact of risk factors for ethnic differences in loneliness. European Journal of Public Health, 26(6), 977-983. https://doi.org/10.1093/eurpub/ckw115

\section{About the Authors}

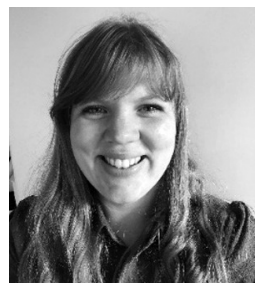

Rowan L. F. ten Kate is a PhD student in sociology at the University of Groningen (RUG). After completing the research master behavioural and social sciences at RUG, Rowan continues to study research topics related to social networks, social support, and social wellbeing in her PhD research. Her doctoral thesis focuses on feelings of loneliness and social relationships of older migrants living in Northwestern Europe. 


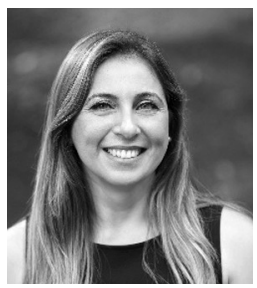

Başak Bilecen is an associate professor of sociology and Rosalind Franklin Fellow at the University of Groningen. Her research focuses on international migration, transnational social protection, social inequalities, social networks and well-being. She is the author of International Student Mobility and Transnational Friendships (Palgrave, 2014) and has co-edited special issues in such journals as the Comparative Migration Studies (2019), Social Networks (2018), Journal of Ethnic and Migration Studies (2017), and Population, Space and Place (2015).

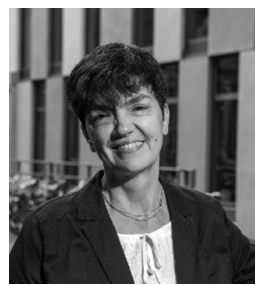

Nardi Steverink is a professor of sociology at the University of Groningen and the University Medical Centre Groningen, and programme leader of the "Self-Management of Well-Being" intervention. This intervention focuses on the improvement of self-management ability, subjective well-being, and loneliness. In her research line "Aging Well: Social Relations, Self-Regulation, Health and Well-Being," Nardi focuses on questions concerning social and behavioural determinants of health, well-being, and quality of life over the lifespan, with a special focus on the role of social needs and self-regulation. 